\title{
Prevalência de alto risco cardiovascular na população adulta brasileira segundo diferentes critérios: estudo comparativo
}

\author{
Prevalence of high risk for cardiovascular disease among the \\ Brazilian adult population, according to different risk calculators: \\ a comparative study
}

Deborah Carvalho Malta (http://orcid.org/0000-0002-8214-5734) ${ }^{1}$

Pedro Cisalpino Pinheiro (http://orcid.org/0000-0002-6954-1708) ${ }^{1}$

Renato Teixeira Azeredo (http://orcid.org/0000-0002-1259-6812) ${ }^{1}$

Filipe Malta Santos (https://orcid.org/0000-0002-0522-0374) ${ }^{1}$

Antonio Luiz Pinho Ribeiro (http://orcid.org/0000-0002-2740-0042) ${ }^{1}$

Luisa Campos Caldeira Brant (https://orcid.org/0000-0002-7317-1367) ${ }^{1}$

${ }^{1}$ Faculdade de Medicina, Universidade Federal de Minas Gerais. Av. Alfredo Balena 190, Santa Efigênia. 30130-100 Belo Horizonte MG Brasil.

dcmalta@uol.com.br

\begin{abstract}
This study compares the proportion of the Brazilian adult population classified as being at high risk of cardiovascular disease (CVD) based on six different $C V D$ risk calculators in order to assess the agreement across different tools. A cross-sectional study was conducted using laboratory data from the National Health Survey (NHS). The prevalence rates of high 10-year risk of CVD among individuals aged between 45 and 64 years were as follows: Brazilian Society of Cardiology (BSC) global risk score (GRS) - 38.1\%; American College of Cardiology/American Heart Association (ACC/AHA) score - 44.1\%; Framingham Heart Study/GRS - 19.4\%; European Society of Cardiology SCORE - 14.6\%; World Health Organization/International Society of Hypertension (WHO/ISH) score - 3.1\%; and Lim et al. $-2.5 \%$. The CVD calculators showed poor agreement for the identification of high-risk individuals and a high level of agreement for the identification of low/moderate risk individuals, except for the ACC/AHA risk score. The findings show that the proportion of individuals classified as eligible for preventive drug therapy varies from tool to tool, which could lead to the misinterpretation of risk, poor cost-effectiveness of therapy and difficulty implementing public policies.
\end{abstract}

Key words Cardiovascular diseases, Risk factors, Prevalence, Brazil
Resumo O estudo visa comparar a proporção de indivíduos classificados como portadores de alto risco cardiovascular $(R C V)$ na população adulta brasileira, segundo seis diferentes calculadoras de risco, visando analisar a concordância entre as medidas. Estudo transversal, no qual foram utilizados dados laboratoriais da Pesquisa Nacional de Saúde (PNS). As prevalências do RCV em 10 anos para a população entre 45 e 64 anos foram: Escore de risco global (ERG) da Sociedade Geral de Cardiologia (SBC):38,1\%, "American College of Cardiology" e "American Heart Association" ACC/AHA, 44,1\%, "Framingham Heart Stu$d y$ "/ERG 19,4\%, SCORE da "European Society of Cardiology", 14,6, Organização Mundial da Saúde/Sociedade Internacional de hipertensão (OMS/ISH) e Lim et al. As calculadoras de RCV apresentaram baixa concordância para identificar os indivíduos de alto risco e alta concordância dos de risco baixo/moderado, exceto pela ACC/AHA. O emprego de diferentes calculadoras resultou em diferentes populações elegíveis para iniciar a terapia farmacológica para prevenção cardiovascular, o que pode implicar em percepções de risco inadequadas, baixo custo efetividade desse tratamento e dificuldade de implementação de políticas públicas.

Palavras-chave Doenças cardiovasculares, Fatores de risco, Prevalência, Brasil 


\section{Introdução}

As doenças cardiovasculares (DCV) foram responsáveis por aproximadamente 18 milhões de mortes em 2016, sendo que aproximadamente $80 \%$ desses óbitos ocorreram em países de baixa e de média renda ${ }^{1}$. Associam-se a essas doenças, condições socioeconômicas desfavoráveis, como pobreza, baixa escolaridade e renda ${ }^{2,3}$, além da urbanização acelerada, aumento da expectativa de vida ${ }^{1-3}$, fatores de risco (FR) comportamentais (uso de tabaco, uso de álcool, dieta inadequada, sedentarismo) e metabólicos (obesidade, glicemia elevada, pressão arterial elevada, hiperlipidemia $)^{1-4}$. Sabe-se que os FR, quando agregados, resultam em aumento do risco de eventos cardiovasculares adversos (RCV) que se sobrepõe a cada FR individualmente, sendo prioritária a detecção e a abordagem precoce dessa população de alto RCV ${ }^{1,4}$.

Assim, a Organização Mundial da Saúde (OMS) preconiza políticas públicas de âmbito populacional para estimular a promoção da saúde, a prevenção primordial e primária, com medidas regulatórias como a taxação de tabaco, álcool e alimentos ultraprocessados ${ }^{5,6}$, com a criação de ambientes que tornem acessíveis e estimulem as escolhas saudáveis ${ }^{6}$. Ainda, no contexto de prevenção primária, definida nesse caso como a prevenção anterior a qualquer evento cardiovascular, a OMS recomenda a identificação de indivíduos portadores de alto RCV por meio de escores ou calculadoras que estimem o risco agregado desses $\mathrm{FR}^{4}$.

A identificação dos indivíduos de alto RCV permite adicionar às medidas preventivas acima, aconselhamento e tratamento farmacológico específico para esse grupo, como a prescrição de estatinas $^{4}$ ou o tratamento farmacológico ainda no estágio de pré-hipertensão ${ }^{7,8}$. Ambas as ações citadas visam a prevenir mortes e eventos cardiovasculares adversos não fatais, principalmente a doença arterial coronariana (DAC) e o acidente vascular cerebral (AVC), as duas principais causas de morte no Brasili ${ }^{1,9}$. Por esses motivos, as calculadoras de RCV tornaram-se importantes instrumentos de apoio para a saúde pública, em especial para as equipes da Atenção Primária à Saúde (APS), auxiliando nas decisões de aconselhamento e tratamento dos usuários ${ }^{4}$. No entanto, a escolha de qual calculadora de RCV deve ser utilizada no Brasil é motivo de debate, tendo em vista que ainda não existe equação derivada de estudo na população brasileira que considere as características da nossa população como composição racial, condições socioeconômicas e geográficas, valores de referência laboratoriais específicos, entre outras. Com isso pode-se classificar o risco do indivíduo de forma imprecisa.

Diversas calculadoras de RCV foram desenvolvidas e, em geral, diferem-se devido às características da população da qual foram derivadas (sexo, faixa etária, raça), e da presença ou ausência de medidas preventivas para FR utilizadas nessa população, que variaram ao longo da história e das políticas de saúde locais ${ }^{10}$. Apesar dessas calculadoras incluírem FR semelhantes, tais fatores podem diferir e terem pesos diferentes quanto ao RCV, e ainda há diferenças entre as quais os desfechos cardiovasculares que cada calculadora prediz em 10 anos e como esses foram adjudicados ${ }^{10}$. Existem escores que calculam o risco apenas de mortes cardiovasculares, enquanto outros incluem diversos eventos cardiovasculares não fatais. Adicionalmente, cada calculadora tem uma proposta de ponto de corte para classificar os indivíduos como alto RCV, que levam em consideração as características acima e também o risco que aqueles autores consideram aceitáveis para a indicação de estatina, considerando o risco-benefício da medicação, no contexto de cada país ${ }^{11}$.

Desta forma, o objetivo deste estudo foi comparar a proporção de indivíduos classificados como portadores de alto RCV na população brasileira, segundo diferentes calculadoras de risco, visando analisar a concordância entre as medidas. Pretende-se discutir a implicação desses achados para a abordagem preventiva dos indivíduos de alto risco e para o planejamento de políticas públicas em nosso país.

\section{Métodos}

Trata-se de estudo transversal, que utilizou dados da Pesquisa Nacional de Saúde (PNS), um amplo inquérito nacional, realizado pelo Instituto Brasileiro de Geografia e Estatística (IBGE) em parceria com o Ministério da Saúde (MS), em 2013, e completada com subamostra laboratorial em 2014 e 2015, cujos dados serão analisados neste estudo ${ }^{12}$.

Foi selecionada uma subamostra de $25 \%$ dos setores censitários selecionados para a PNS 2013 obedecendo à estratificação da amostra da PNS, com probabilidade inversamente proporcional à dificuldade de coleta ${ }^{11}$. O plano amostral empregado foi a amostragem por conglomerados em três estágios de seleção. As unidades primárias de 
amostragem (UPA) foram os setores censitários, ou a composição de setores, enquanto que, o segundo estágio, foi selecionado por amostragem aleatória simples de um número fixo de domicílios particulares permanentes (10 a 14) em cada UPA, e, no terceiro estágio, um morador com 18 anos ou mais foi selecionado, também aleatoriamente, a partir de uma lista de elegíveis construída no momento da entrevista ${ }^{11}$. Tendo em vista que foram selecionados $25 \%$ dos setores censitários para a realização dos exames laboratoriais e, supondo-se taxa de não resposta de 20,0\%, o número esperado de indivíduos com dados laboratoriais foi de $12 \mathrm{mil}$, aproximadamente ${ }^{11}$. No Brasil, como um todo, os exames bioquímicos foram realizados em 8.952 indivíduos. Outros detalhes podem ser consultados em publicação específica $^{12,13}$, assim como a descrição dos aspectos referentes à coleta laboratorial ${ }^{12,13}$ e aos pontos de corte adotados para estimar a população com diabetes ${ }^{13}$, colesterol alterado ${ }^{14,15}$, aspectos referentes à coleta da pressão arterial $^{16}$, à aferição do tabagismo e à DCV prévia $^{17,18}$.

Os indivíduos foram classificados em alto ou baixo/intermediário $\mathrm{RCV}$, nomeado baixo RCV, por 6 (seis) diferentes critérios, a saber: 1) A recomendação da Sociedade Brasileira de Cardiologia (SBC) proposta em 2019, que segue a calculadora sugerida pelo Framingham Heart Study para o escore de risco global (ERG-FHS) ${ }^{19}$, classificando o indivíduo em alto risco se $>20 \%$ para o sexo masculino e $>10 \%$ para o sexo feminino, acrescentando ainda outras variáveis detalhadas abaixo; 2) Pooled cohort equation, que foi introduzida pelos American College of Cardiology e American Heart Association (ACC/AHA) em 2013 ${ }^{20}$, que utilizou dados de vários estudos de coorte americanos, para derivação e validação de novas equações específicas para sexo e raça ; 3) escore de risco global do Framingham Heart Study (ERG-FHS), de $2008^{21}$; 4) SCORE, calculadora proposta pela European Society of Cardiology desde 2007, que deriva de várias coortes europeias e que propõe duas equações conforme o RCV para as regiões de alto (oriental) e baixo (ocidental) RCV da Europa, das quais escolhemos conservadoramente para esta análise a equação de alto risco, por não sabermos o risco da população brasilei$\left.\mathrm{ra}^{22} ; 5\right)$ Lim et al. ${ }^{23}$, que a partir de modelos de microssimulação construíram uma tabela geral para estimativa do RCV por país; 6) calculadora proposta pela Organização Mundial da Saúde/ Sociedade Internacional de hipertensão OMS/ ISH para suas 14 sub-regiões epidemiológicas, das quais selecionamos aquela que o Brasil se enquadra, denominada América $\mathrm{B}^{24}$ (Tabela 1). Essas calculadoras foram escolhidas por serem as mais utilizadas na prática clínica ou por serem direcionadas ao nosso país ou região geográfica.

É importante notar que a faixa etária dos indivíduos, as variáveis e os desfechos avaliados diferem para cada uma das medidas de risco RCV (calculadoras), assim como o ponto de corte proposto para identificar indivíduos de alto RCV, conforme especificado na Tabela 1.

No que se refere à recomendação da SBC em diretriz de $2019^{19}$, os indivíduos também foram classificados em alto RCV, conforme o ERG ${ }^{21}$ com ponto de corte $>20 \%$ para homens, porém $>10 \%$ para mulheres, ou a presença de outras variáveis, como indivíduos com doença renal crônica (taxa de filtração glomerular $<60 \mathrm{ml} / \mathrm{min}$ e em fase não dialítica); ou com colesterol LDL $\geq 190 \mathrm{mg} / \mathrm{dL}$; ou indivíduos que apresentavam, simultaneamente, diabetes e LDL entre $\geq 70$ e $<$ $190 \mathrm{mg} / \mathrm{dL}$, além de pelo menos um estratificador de risco (homens com idade $\geq 48$ e mulheres $\geq$ 54 ; tempo de diagnóstico de diabetes $\geq 10$ anos; tabagismo; hipertensão arterial sistêmica (pressão sistólica $\geq 140$ ou diastólica $\geq 90 \mathrm{mmHg}$ ), síndrome metabólica de acordo com a Internacional Diabetes Federation ${ }^{28}$ (com a substituição do Triglicérides pelo Colesterol Total, em função da indisponibilidade da primeira na base de dados $)^{12,14}$. A SBC utiliza também na definição de alto RCV os indivíduos portadores de aterosclerose subclínica, aneurisma de aorta abdominal, histórico familiar de parente de primeiro grau com DCV prematura e presença de albuminúria $^{19}$, informações não incluídas nesta análise em função da indisponibilidade na base de dados da PNS.

Em relação às análises estatísticas deste estudo, os participantes com DCV conhecida foram excluídos da análise atual, uma vez que as calculadoras aqui utilizadas assim o fazem. Para permitir comparações entre as diferentes métricas, a análise adicional foi realizada entre os participantes na faixa etária de $45<65$ anos, a qual foi coberta por todas as calculadoras utilizadas ( $\mathrm{n}=$ 2.791). As medidas de risco, cujo resultado era contínuo (SCORE, ACC/AHA) ou com pontuação (ERG), foram analisados de modo binário. Ou seja, aos indivíduos com risco acima de um determinado ponto de corte foi atribuído valor 1 para o alto RCV ou 0, para o baixo RCV. As medidas desenvolvidas por Lim et al. ${ }^{23}$, pela OMS/ $\mathrm{ISH}^{24}$ e pela $\mathrm{SBC}^{19}$ calculam os riscos de modo dicotômico. A partir da classificação dos indivíduos conforme o RCV, em alto ou baixo (prevalência), 
Tabela 1. Características das medidas de risco cardiovascular analisadas, segundo faixa etária, variáveis utilizadas, desfechos avaliados em 10 anos e pontos de corte propostos para classificação dos indivíduos em alto risco cardiovascular

\begin{tabular}{|c|c|c|c|c|}
\hline $\begin{array}{l}\text { Calculadora } \\
\text { de RCV }\end{array}$ & $\begin{array}{l}\text { Faixa } \\
\text { etária }\end{array}$ & Variáveis utilizadas & $\begin{array}{c}\text { Desfechos avaliados em } 10 \\
\text { anos }\end{array}$ & $\begin{array}{l}\text { Ponto de } \\
\text { corte }\end{array}$ \\
\hline $\mathrm{ACC} \mathrm{AHA}^{20}$ & $40-79$ & $\begin{array}{l}\text { Idade, sexo, PAS, uso de } \\
\text { antihipertensivo, CT, HDL-C, DM, } \\
\text { tabagismo }\end{array}$ & $\begin{array}{l}\text { Doença coronariana fatal, } \\
\text { IAM não fatal e AVC fatal ou } \\
\text { não fatal }\end{array}$ & $\geq 7,5 \%$ \\
\hline $\begin{array}{l}\text { Framingham } \\
(\mathrm{ERG})^{21}\end{array}$ & $30-74$ & $\begin{array}{l}\text { Idade, sexo, PAS, uso de } \\
\text { antihipertensivo, CT, HDL-C, DM, } \\
\text { tabagismo }\end{array}$ & $\begin{array}{l}\text { Doença cardiovascular } \\
\text { fatal e não fatal (coronária, } \\
\text { cerebrovascular, insuficiência } \\
\text { cardíaca, claudicação } \\
\text { intermitente) }\end{array}$ & $\geq 20 \%$ \\
\hline $\begin{array}{l}\text { ERG- } \\
\text { Recomendação } \\
\text { da } \mathrm{SBC}^{19}\end{array}$ & $30-74$ & $\begin{array}{l}\text { Idem ERG ou Aterosclerose } \\
\text { subclínica }{ }^{\star} \text { ou Aneurisma de aorta } \\
\text { abdominal ou Colesterol LDL-c } \\
\geq 190 \mathrm{mg} / \mathrm{dL} \text { ou Doença Renal } \\
\text { Crônica ou Diabetes com LDL } 70 \text { - } \\
\text { 190mg/dL e ER }{ }^{\star \star} \text {; DASC }\end{array}$ & $\begin{array}{l}\text { Doença cardiovascular } \\
\text { fatal e não fatal (coronária, } \\
\text { cerebrovascular, insuficiência } \\
\text { cardíaca, claudicação } \\
\text { intermitente) }\end{array}$ & $\begin{array}{l}\geq 10 \% \\
\text { Mulheres } \\
\mathrm{e} \geq 20 \% \\
\text { homens; } \\
\text { mais outros } \\
\text { critérios }\end{array}$ \\
\hline OMS/ISH ${ }^{24}$ & $40-79$ & $\begin{array}{l}\text { Idade, sexo, PAS, CT, DM, } \\
\text { tabagismo }\end{array}$ & $\begin{array}{l}\text { IAM ou AVC (fatal ou não } \\
\text { fatal) }\end{array}$ & $\geq 20 \%$ \\
\hline Lim et al. ${ }^{23}$ & $40-79$ & Idade, sexo, PAS, BMI, tabagismo & $\begin{array}{l}\text { Doença coronariana ou AVC } \\
\text { (fatal) }\end{array}$ & $\geq 15 \%$ \\
\hline $\begin{array}{l}\text { SCORE (Alto } \\
\text { Risco }-\mathrm{CT})^{22}\end{array}$ & $45-64$ & Idade, sexo, PAS, CT, tabagismo & $\begin{array}{l}\text { Morte cardiovascular } \\
\text { (coronariana, AVC, arritmia, } \\
\text { aneurisma de aorta ou } \\
\text { doença vascular periférica) }\end{array}$ & $\geq 5 \%$ \\
\hline
\end{tabular}

AVC: acidente vascular cerebral, CT: colesterol total, DM: diabetes mellitus, HDL-c: lipoproteína de alta densidade, IAM: infarto agudo do miocárdio, PAS: pressão arterial sistólica. LDL, ACC/AHA- Colégio Americano de Cardiologia/American Heart Association ${ }^{20} \mathrm{OMS} / \mathrm{ISH}$ - Organização Mundial da Saúde /a Sociedade Internacional de Hipertensão ${ }^{24}$; Escore de risco global (ERG) da Sociedade Brasileira de Cardiologia. SBC ${ }^{19}$ - Framingham Heart Study (ERG $)^{21}$, SCORE da European Society of Cardiolog $y^{22}$.

*Apesar de fazer parte da recomendação da SBC, a aterosclerose subclínica não foi incorporada na presente análise por ausência de dados. ${ }^{\star *}$ Estratificadores de Risco: Idade $\geq 48$ anos no homem e $\geq 54$ anos na mulher; tempo de diagnóstico do diabetes $>10$ anos; histórico familiar de parente de primeiro grau com DCV prematura ( $<55$ anos para homens e $<65$ anos para mulher); tabagismo (pelo menos um cigarro no último mês); hipertensão arterial sistêmica; síndrome metabólica (SM), de acordo com a International Diabetes Federation; presença de albuminúria $>30 \mathrm{mg} / \mathrm{g}$ de creatinina e/ou retinopatia; taxa de filtração glomerular $<60 \mathrm{~mL} / \mathrm{min}$. *** DASC: Doença Aterosclerótica Subclínica: Ultrassonografia de carótidas com presença de placa $>1,5 \mathrm{~mm}$; ITB $<0,9$; escore de cálcio coronário > 10 unidades Agatston; presença de placas ateroscleróticas na angiotomografia de coronárias; LDL-c entre 70 e 189 $\mathrm{mg} / \mathrm{dL}$, com escore de risco global do sexo masculino $>20 \%$ e $>10 \%$ para o sexo feminino.

a concordância entre as calculadoras foi avaliada, utilizando a porcentagem de concordância par a par, empregando, como referência, o modelo proposto para a classificação de indivíduos em alto risco pela $\mathrm{SBC}^{19}$, visto que é a recomendação contemporânea disponível em nosso país. A porcentagem de concordância mede a proporção daqueles com RCV alto ou baixo pela calculadora em questão e pela recomendação da SBC.

A PNS foi aprovada Comissão Nacional de Ética em Pesquisa (CONEP) do Ministério da Saúde ${ }^{17}$.

\section{Resultados}

A Figura 1 mostra a prevalência de alto RCV em 10 anos para a população entre 45 e 64 anos, utilizando os pontos de corte sugeridos por cada uma das calculadoras. A prevalência de RCV mais elevada foi observada na medida desenvolvida pela ACC/AHA, 44,1\% (IC95\%, 41,7-46,5). A métrica proposta pela $\mathrm{SBC}$ apresentou a segunda maior prevalência de alto RCV, 38,1\% (IC95\%, 35,840,4). A prevalência de RCV elevado, pela ERG, foi de 19,4\% (IC95\%, 17,5-21,4). A quarta maior 


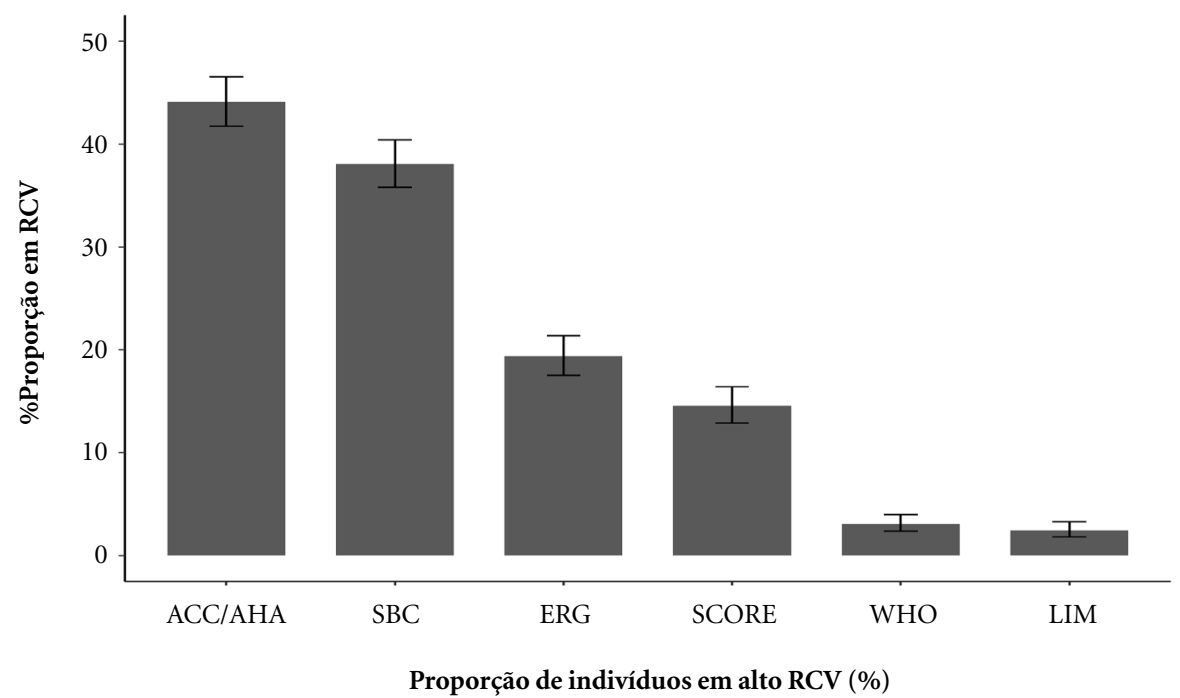

Figura 1. Proporção da população entre 45 e 64 anos com alto risco cardiovascular (RCV) conforme diferentes calculadoras de risco cardiovascular, Brasil. Pesquisa Nacional de Saúde - 2013, 2014-2015.

prevalência de alto RCV foi estimada com base no SCORE, 14,6\% (IC95\%, 12,9-16,4). As prevalências de alto RCV mais baixas foram estimadas com base na métrica da OMS/ISH ${ }^{24}$ e na medida desenvolvida por Lim et al. ${ }^{23}$, respectivamente, 3,1\% (IC95\%, 2,4-4) e 2,5\% (IC95\%, 1,8-3,3).

A Figura 2 mostra as prevalências de alto RCV, também utilizando os pontos de corte recomendados pelas calculadoras, mas empregando o intervalo etário proposto pelas mesmas. Ou seja, neste caso, a população na qual o RCV foi avaliado varia conforme a idade. A maior prevalência de alto risco foi mais uma vez observada na métrica da ACC/AHA, 39,4\% (IC95\%, 37,6-41,3), nesse caso, considerando-se a população entre 40 e 79 anos. A proposta da SBC manteve-se como a segunda maior prevalência, considerando o grupo etário recomendado (30-74), 28,8\% (IC95\%, 27,4-30,2). A ERG apresentou prevalência de alto RCV (14,7\%, IC95\% 13,6-15,9) muito próxima da estimada pelo SCORE (14,6\%, IC95\% 12,9$16,4)$. A primeira, no entanto, considera um intervalo etário mais extenso (30-74) que a segunda (45-64). Outra vez, a medida de Lim et al. ${ }^{23}$ e da OMS/ISH ${ }^{24}$ apresentaram as prevalências mais baixas.

A Tabela 2, além das prevalências de indivíduos com alto RCV de cada calculadora, representadas na Figura 1, mostra a porcentagem de concordância entre cada uma das medidas em relação à recomendação da SBC para estimativa de alto e baixo risco para os indivíduos entre 45 e 64 anos. A maior concordância observada para o grupo de alto risco foi entre a ERG e a SBC, 50,9\% (IC95\%, 47,1-54.7), já que a primeira faz parte do cálculo da recomendação da SBC. Verifica-se que 43,5\% (IC95\%, 39,7-47,3) daqueles indivíduos de alto RCV, pela métrica da SBC, foram considerados em elevado RCV pela medida recomendada pela ACC/AHA, enquanto que pelo SCORE esse número foi 29,4\% (IC95\% 26-33,1). Já em relação à métrica da OMS e a de Lim et al. ${ }^{23}$, menos de $10 \%$ daqueles com alto RCV, com base na recomendação da SBC, foram classificados como alto RCV por elas, sendo as mais baixas concordâncias verificadas.

Quando comparada a porcentagem de concordância para o grupo de baixo risco das métricas em relação àquela recomendada pela SBC, a concordância mais baixa foi observada para a ACC/AHA. Ou seja, 55,5\% daqueles que não faziam parte do alto RCV da SBC, também não foram considerados como de alto RCV pela medida da ACC/AHA. O ERG apresentou 100\% de concordância para o baixo risco, dado esperado já que a medida da SBC utiliza o ERG, porém com pontos de corte mais baixos, além de incluir outras categorias em sua definição de alto risco. 


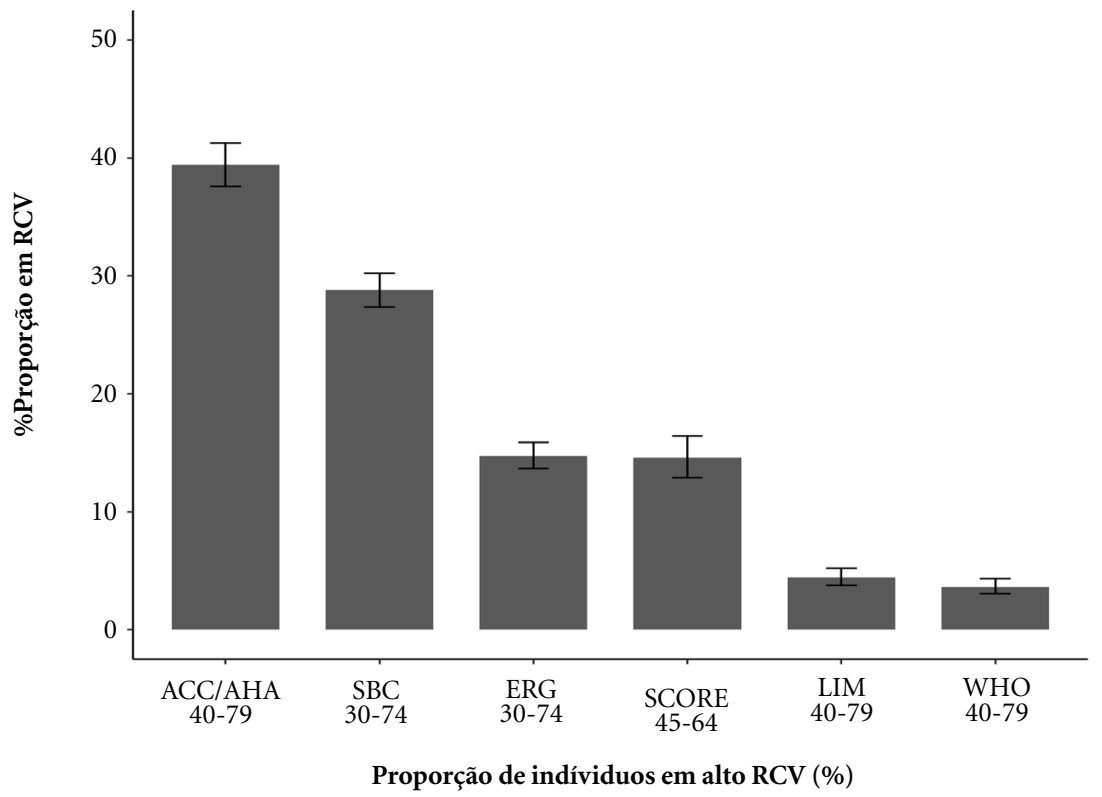

Figura 2. Proporção da população com alto risco cardiovascular (RCV), considerando grupos etários e pontos de corte propostos pelas diferentes calculadoras de risco cardiovascular, Brasil. Pesquisa Nacional de Saúde - 2013, 2014-2015.

Tabela 2. Porcentagens de concordância entre as seis calculadoras de risco cardiovascular para classificação de indivíduos em alto ou baixo risco cardiovascular, utilizando como referência a proporção calculada conforme recomendação da Sociedade Brasileira de Cardiologia (SBC), Brasil. Pesquisa Nacional de Saúde, 2014-2015.

\begin{tabular}{lrcc}
\hline \multicolumn{1}{c}{ Calculadora de RCV } & $\begin{array}{c}\text { Prevalência } \\
\text { \% (IC 95\%) }\end{array}$ & $\begin{array}{c}\text { Concordância para alto } \\
\text { risco (\%) }\end{array}$ & $\begin{array}{c}\text { Concordância para } \\
\text { baixo risco (\%) }\end{array}$ \\
\hline SBC & $38,1(35,8 ; 40,4)$ & referência & referência \\
ACC/AHA & $44,1(41,7 ; 46,5)$ & $43,5(39,7 ; 47,3)$ & $55,5(52,4 ; 58,6)$ \\
ERG (FHS) & $19,4(17,5 ; 21,4)$ & $50,9(47,1 ; 54,7)$ & $100(100 ; 100)$ \\
WHO & $3,1(2,4 ; 4)$ & $7,6(5,8 ; 9,9)$ & $99,7(99,3 ; 99,8)$ \\
LIM & $2,5(1,8 ; 3,3)$ & $5,8(4,2 ; 7,9)$ & $99,6(99,2 ; 99,8)$ \\
SCORE & $14,6(12,9 ; 16,4)$ & $29,4(26 ; 33,1)$ & $94,6(92,8 ; 95,9)$ \\
\hline
\end{tabular}

Fonte: Pesquisa Nacional de Saúde 2013, 2014-2015.

Em relação ao SCORE, a porcentagem de concordância para o grupo de baixo risco foi de $94,6 \%$ (IC95\%, 92,8-95,9), enquanto que para a métrica da OMS e a de Lim et al. ${ }^{23}$ foi superior a $99 \%$.

\section{Discussão}

Este estudo analisou a proporção de indivíduos classificados como alto RCV, conforme seis diferentes escores de risco, utilizando a base de dados laboratoriais da PNS - amostra representativa da população adulta brasileira. Tendo como referência para a classificação do RCV a recomendação 
da SBC, em geral, as outras calculadoras de RCV apresentaram baixa concordância para detectar os indivíduos de alto RCV, e alta concordância para identificar indivíduos de baixo RCV, exceto a calculadora da ACC/AHA. Além disso, o estudo apontou que os instrumentos de avaliação de RCV mais utilizados atualmente apresentaram grande variação na proporção dos indivíduos classificados como alto RCV, podendo classificar até $39 \%$ da população de 45 a 65 anos como alto RCV. Sendo assim, dependendo do instrumento adotado, diferentes populações seriam elegíveis para iniciar terapia farmacológica para prevenção cardiovascular, o que pode implicar em percepções de risco inadequadas, baixa relação de custo-efetividade desse tratamento e dificuldade de implementação de políticas públicas.

A baixa concordância para a identificação de indivíduos de alto RCV aqui reportadas já foram descritas na literatura. Allan et al. ${ }^{25}$, em 2013, utilizaram dados hipotéticos aplicados a 25 calculadoras de risco diferentes e também encontraram concordância ruim entre os modelos, da ordem de $67 \%$, destacando a necessidade de calibrar os modelos de avaliação de RCV para populações específicas, visando a implementação efetiva de diretrizes clínicas para uma terapia farmacológica preventiva ${ }^{24}$. Em geral, escores de risco já foram desenvolvidos ou calibrados para populações dos EUA e da Europa, faltando estudos em países de baixa e média renda, onde fatores modificadores socioeconômicos, de acesso à saúde, racial e cultural podem ter implicações ${ }^{10}$.

Outros estudos alertam também para um risco superestimado de eventos, especialmente da calculadora da ACC/AHA. DeFilippis et al. ${ }^{26}$, em 2015, encontraram discordância entre o que foi predito pelas calculadoras derivadas do Framingham Risk Score ${ }^{21}$ e da ACC/AHA ${ }^{20}$ e os eventos observados, da ordem de 37 a $154 \%$, em 4.000 pacientes do sexo masculino acompanhados em estudo prospectivo de 2000 a 2010 nos EUA. Os autores sugerem que tais alterações podem ser decorrentes principalmente do uso de coortes antigas na elaboração das calculadoras, com provável mudança do perfil populacional, melhora terapêutica durante o estudo e identificação de fatores de risco nos últimos anos, além da dificuldade de avaliar alguns dos fatores de risco como a quantidade de uso de cigarro ou álcool, ou de limitações do próprio estudo de conseguir identificar esses eventos. Outros estudos europeus, como o de Kavousi et al. ${ }^{27}$, em 2014, também observaram riscos superestimados em pacientes de Roterdã, em coorte de 1997-2001 a 2012, em pacientes sem eventos cardiovasculares prévios maiores de 55 anos, chamando a atenção de que a maior parte dos homens do estudo e $65 \%$ das mulheres seria elegível para o tratamento com estatinas pela calculadora ACC/AHA.

No contexto latino americano, em uma análise realizada em Honduras ${ }^{28}$, país considerado de baixa e média renda, na qual foram calculadas as estimativas de RCV, considerando quatro calculadoras distintas, os resultados mostraram proporção elevada de indivíduos de alto RCV, de acordo com os escores avaliados. Para homens e mulheres, indivíduos de alto RCV pelo escore proposto pela ACC/AHA, foi $62,0 \%$ e $29,8 \%$, respectivamente, enquanto que pelo ERG foi $46,1 \%$ e 15\%, e pelo MESA Risk Score foi 70,6\% e 17,7\%, respectivamente ${ }^{28}$. Outro estudo ${ }^{10}$, realizado com 2.183 indivíduos de diferentes regiões do Peru, avaliou a concordância entre sete escores de RCV e verificou, de forma semelhante aos nossos achados, que a concordância entre os escores foi baixa e a variação da proporção de indivíduos considerados de alto RCV foi alta, de $29 \%$ (16,9$31,0 \%$ ), pelo escore proposto pela ACC/AHA, a $0,6 \%(0,2-8,6 \%)$, para o escore proposto pela OMS. Ainda, de acordo com o referido estudo ${ }^{10}$, os autores concluíram que existem incertezas em relação a qual calculadora de RCV utilizar no Peru e em países de baixa e média renda; argumento corroborado pelos resultados da presente pesquisa.

A baixa concordância entre os escores para detectar indivíduos de alto RCV, como já citado, pode estar relacionada aos diferentes desfechos incluídos em cada escore e, também, devido a pressupostos de fatores de riscos aferidos em diversas populações, que na maioria das vezes encontram-se disponíveis em países desenvolvidos, que têm conduzido estudos longitudinais com maior frequência. Outros fatores podem ser a própria mudança das tendências dos FR e de tratamento dos mesmos na população local. Tais situações demandam estudos de prevalência, monitoramento mediante coleta de medidas bioquímicas, antropométricas e físicas para aferir as tendências ${ }^{10}$.

O benefício das estatinas tem sido destacado na prevenção cardiovascular em indivíduos de alto RCV, conforme estudo de meta-análise ${ }^{9}$, que destaca a redução da mortalidade em eventos vasculares maiores. Esta experiência, aliada a um perfil seguro e à redução do custo dos medicamentos, permitiu indicações mais permissivas em alguns países ${ }^{9}$. Entretanto, ainda existem divergências quanto à definição do ponto de corte 
de cada escore de risco, para classificar o alto RCV. Nestas definições dos escores são considerados o risco-benefício das estatinas?. Tradicionalmente, no ERG, o indivíduo era classificado como alto RVC se o risco estimado fosse $>20 \%$. Em 2013, o novo escore proposto pela ACC/AHA ${ }^{28}$, por exemplo, considera apenas Infarto Agudo do Miocárdio e Acidente Vascular Cerebral como fatais e não fatais, mas reduziu o ponto de corte para $>7,5 \%{ }^{28}$, aumentando a proporção da população classificada como alto RCV. Alguns autores criticam a redução do ponto de corte devido à consequente medicalização, a um custo adicional não prioritário para determinados países, particularmente aqueles com recursos limitados ${ }^{29}$. No Brasil, estudo de custo-efetividade ${ }^{30}$ sugere que o uso de estatina de dose intermediária é custo-efetivo para pacientes de alto RCV, considerando o escore ERG e o ponto de corte de $20 \%$, porém não sabemos se o mesmo é verdadeiro com base em escores que resultam em prescrição mais permissiva das estatinas. Além disto, devem ser considerados os recursos financeiros disponíveis para ampliar o tratamento em larga escala.

Independente de qual escore seja utilizado para avaliar o RCV de um indivíduo, é importante enfatizar que os próprios escores de RCV têm limitações, já que, de forma geral, avaliam o risco em 10 anos - o que pode subestimar o risco ao longo da vida ${ }^{28}$-, não incluem fatores de risco mais proximais, como a condição socioeconômi$\mathrm{ca}^{31}$ - que está relacionada ao acesso à saúde de qualidade - e à localização geográfica, ou, ainda, fatores modificadores que aumentam o RCV, que devem ser analisados individualmente, como: história familiar para DCV prematura ${ }^{31}$, hipercolesterolemia familiar ${ }^{32}$, doença renal crônica, doenças inflamatórias, carga tabágica ${ }^{33}$, entre outros. Portanto, para a aplicação adequada na prática clínica, faz-se necessário também individualizar o tratamento e avaliar criteriosamente o $\mathrm{RCV}$ calculado. Ademais, mesmo que o aumento do RCV resultante de determinados FR, como o diabetes e a hipertensão arterial sistêmica, seja gradual e possa ser reduzido por tratamento, ele é avaliado em alguns dos escores de forma dicotômica, além de alguns não considerarem o uso de tratamento para esses FR como variáveis no modelo de predição de risco ${ }^{33}$.

Enquanto não tivermos uma calculadora específica desenvolvida para a população brasileira, precisaremos ainda utilizar uma das propostas citadas para calcular o RCV do indivíduo e avaliar a elegibilidade para prevenção primária farmacológica, conforme recomendação da OMS ${ }^{4}$. Po- rém, ao selecionar a calculadora, deve-se ampliar o conhecimento sobre as diferenças entre elas nos fatores de risco e nos desfechos avaliados, assim como selecionar o ponto de corte para classificar o alto RCV. Além disso, é preciso considerar que a concordância entre elas para identificar os indivíduos de alto risco é baixa, enquanto que a concordância para detectar indivíduos de baixo risco é alta - exceto pela calculadora proposta pela AHA/ACC, por ser mais permissiva para o uso da estatina, ao reduzir o ponto de corte dos pacientes de alto RCV para $>7,5 \%{ }^{21}$. Ou seja, ao identificar um indivíduo como portador de baixo RCV, pela maioria das calculadoras, ele será realmente baixo $\mathrm{RCV}$, porém, ao identificar um indivíduo como alto RCV, será necessária uma avaliação mais criteriosa para introduzir a terapia farmacológica.

Este estudo apresentou como limitações: nem todas as equações das calculadoras utilizadas estavam disponíveis e as pontuações baseadas em gráficos não fornecem avaliações de risco de modo contínuo; a comparabilidade pode ser afetada por diferenças nas definições usadas nos preditores e nos desfechos dos escores de RCV utilizados, conforme demonstrado na Tabela 1 . Porém, reconhecemos tais diferenças e o nosso objetivo foi justamente comparar as calculadoras como são propostas e conforme estão recomendadas nas diretrizes brasileiras ou, aquelas que são aplicadas na prática clínica, para mostrar o quão importante é entender as definições utilizadas nos escores e suas limitações. Ainda, consideramos que a maioria dos profissionais de saúde que aplicam os escores na prática clínica não estão necessariamente cientes dos detalhes técnicos e de modelagem por trás de um rótulo de " 10 anos de alto RCV", com a potencial consequência da introdução de tratamento farmacológico para uma prevenção cardiovascular pouco efetiva.

Enquanto dúvidas pairam no cotidiano da prática clínica para identificar os indivíduos que devam receber terapia farmacológica para prevenir as doenças cardiovasculares, estratégias de prevenção cardiovascular não farmacológicas que estimulem hábitos de vida saudáveis, como cessação do tabagismo, dieta saudável, praticar atividades físicas regularmente e reduzir o uso de álcool -, e de caráter populacional - que independem do risco basal individual - são benéficas e devem ser implementadas de forma abrangente para reduzir a morbimortalidade das doenças cardiovasculares ${ }^{34}$. Já em relação às medidas preventivas específicas para a população de alto risco, torna-se urgente a derivação de uma calcu- 
ladora de RCV brasileira, com dados de coortes nacionais, ou a validação de calculadoras internacionais, que identifiquem corretamente essa população para o tratamento efetivo dos indivíduos de alto RCV e para o planejamento de políticas públicas. Destaca-se ainda a importância de avançar em termos dos valores de referencias dos exames laboratoriais brasileiros, a partir de estudos nacionais, dentro das condições étnicas e socio-culturais locais, estabelecendo pontos de cortes definidos, conforme proposta do laboratório da Pesquisa Nacional de Saúde ${ }^{35}$.

\section{Conclusão}

Este estudo analisou a proporção de indivíduos classificados como alto RCV conforme seis di- ferentes escores de RCV, na subamostra populacional da PNS, que é representativa da população adulta brasileira. Utilizando como referência para a classificação do RCV a recomendação da SBC, as outras calculadoras de RCV, em geral, apresentaram baixa sensibilidade e alta especificidade para detectar os indivíduos de alto RCV. Além disso, o estudo apontou que os escores de RCV mais utilizados atualmente apresentaram grande variação na proporção de indivíduos classificados como alto RCV. Sendo assim, enquanto não tivermos um escore de risco, derivado ou validado para a população brasileira, a prática de generalização das equações de risco e de definição do limiar para o tratamento farmacológico em função das calculadoras de RCV, devem ser rediscutidas em cada contexto e devem considerar o custo/efetividade das recomendações.

\section{Colaboradores}

DC Malta participou da concepção do estudo, planejamento, delineamento, interpretação das análises estatísticas e dos resultados, redação do artigo, revisão crítica do artigo. PC Pinheiro participou do planejamento, análises estatísticas, redação dos resultados e revisão crítica do artigo. FM Santos e LCC Brant participaram da interpretação dos resultados, redação do artigo, revisão crítica do artigo. ALP Ribeiro participou da interpretação dos resultados e revisão crítica do artigo.

\section{Agradecimentos}

Ao Conselho Nacional de Desenvolvimento Científico e Tecnológico ( $\mathrm{CNPq}$ ), pela bolsa de Produtividade em Pesquisa recebida pelas autoras DC Malta e ALP Ribeiro.

À Secretaria de Vigilância em Saúde pelo financiamento do Ministério Saúde. 


\section{Referências}

1. World Health Organization (WHO). Noncommunicable diseases country profiles 2018. Genebra: WHO; 2018.

2. Souza MFM, Malta DC, França EB, Barreto ML. Transição da saúde e da doença no Brasil e nas Unidades Federadas durante os 30 anos do Sistema Único de Saúde. Cien Saude Colet 2018; 23(6):1737-1750.

3. Harper S, Lynch J, Smith GD. Social determinants and the decline of cardiovascular diseases: understanding the links. Annu Rev Public Health 2011; 32:39-69.

4. World Health Organization (WHO). Global NCD target prevent heart attacks and strokes through drug therapy and counselling. Genebra: WHO; 2016.

5. World Health Organization (WHO). "Best Buys" Tackling NCDs: Best buys and other recommended interventions for the prevention and control of noncommunicable diseases. Genebra: WHO; 2017.

6. World Health Organization (WHO). Global action plan for the prevention and control of noncommunicable diseases 2013-2020. Genebra: WHO; 2013.

7. Whelton PK, Carey RM, Aronow WS, Casey Junior DE, Collins KJ, Himmelfarb CD, DePalma SM, Gidding S, Jamerson KA, Jones DW, MacLaughlin EJ, Muntner P, Ovbiagele B, Smith Junior SC, Spencer CC, Stafford RS, Taler ST, Thomas RJ, Williams KA, Williamson JD, Wright Junior JT. ACC/AHA/ AAPA/ABC/ACPM/AGS/APhA/ASH/ASPC/NMA/ PCNA Guideline for the Prevention, Detection, Evaluation, and Management of High Blood Pressure in Adults: Executive Summary: A Report of the American College of Cardiology/American Heart Association Task Force on Clinical Practice Guidelines. Hypertension 2018; 71(6):1269-1324.

8. Malachias MVB, Souza WKSB, Plavnik FL, Rodrigues CIS, Brandão AA, Neves MFT, Bortolotto LA, Franco RJS, Poli-de-Figueiredo CE; Sociedade Brasileira de Cardiologia. 7a Diretriz Brasileira de Hipertensão Arterial. Arquivos Brasileiros de Cardiologia 2016; 107(Supl. 3):1-82.

9. Taylor F, Huffman MD, Macedo AF, Moore THM, Burke M, Smith GD, Ward K, Ebrahim S. Statins for the primary prevention of cardiovascular disease. Cochrane Database Syst Rev 2013; (1):CD004816.

10. Bazo-Alvarez JC, Quispe R, Peralta F, Poterico JA, Valle GA, Burroughs M, Pillay T, Gilman RH, Checkley W, Malaga G, Smeeth L, Bernabé-Ortiz A, Miranda JJ, Peru Migrant Study; CRONICAS Cohort Study Group. Agreement Between Cardiovascular Disease Risk Scores in Resource-Limited Settings: Evidence from 5 Peruvian Sites. Crit Pathw Cardiol 2015; 14(2):74-80.

11. WHO CVD Risk Chart Working Group. World Health Organization cardiovascular disease risk charts: revised models to estimate risk in 21 global regions. Lancet Glob Health 2019; 7(10):E1332-E1345.

12. Szwarcwald CL, Malta DC, Souza Júnior PRB, Almeida WS, Damacena GN, Pereira CA, Rosenfeld LG. Exames laboratoriais da Pesquisa Nacional de Saúde: metodologia de amostragem, coleta e análise dos dados. Rev Bras Epidemiol 2019; 22(Supl. 2):E190004.
13. Malta DC, Duncan BB, Schmidt MI, Machado ÍE, Silva AG, Bernal RTI, Pereira CA, Damacena GN, Stopa SR, Rosenfeld LG, Szwarcwald CL. Prevalência de diabetes mellitus determinada pela hemoglobina glicada na população adulta brasileira, Pesquisa Nacional de Saúde. Rev Bras Epidemiol 2019; 22(Supl 2):E190006.

14. Malta DC, Szwarcwald CL, Machado IE, Pereira CA, Figueiredo AW, Sá ACMGN, Velasquez-Melendez G, Santos FM, Souza-Júnior PRB, Stopa SR, Rosenfeld LG. Prevalência de colesterol total e frações alterados na população adulta brasileira: Pesquisa Nacional de Saúde. Rev Bras Epidemiol 2019; 22(Supl. 2):1-13.

15. American medical association. Executive summary of the Third Report of The National Cholesterol Education Program (NCEP) Expert Panel on Detection, Evaluation, and Treatment of High Blood Cholesterol in Adults (Adult Treatment Panel III). JAMA 2001; 285(19):2486-2497.

16. Malta DC, Santos NB, Perillo RD, Szwarcwald CL. Prevalence of high blood pressure measured in the Brazilian population, National Health Survey, 2013. São Paulo Med J 2016; 134(2):163-170.

17. Instituto Brasileiro de Geografia e Estatística (IBGE). Pesquisa Nacional de Saúde 2013: percepção do estado de saúde, estilos de vida e doenças crônicas: Brasil, Grandes Regiões e Unidades da Federação. Rio de Janeiro: IBGE; 2014.

18. Gonçalves RPF, Haikal DS, Freitas MIF, Machado IE, Malta DC. Diagnóstico médico autorreferido de doença cardíaca e fatores de risco associados: Pesquisa Nacional de Saúde. Rev Bras Epidemiol. 2019; 22(Supl. 2):E190016.

19. Précoma DB, Oliveira GMM, Simão AS, Dutra OP, Coelho OR, Izar MCO, Póvoa RMS, Giuliano ICB, Alencar Filho AC, Machado CV, Scherr C, Fonseca FAH, Santos Filho RD, Carvalho T, Avezum Jr. A, Esporcatte R, Nascimento BR, Brasil DP, Soares GP, Villela PB, Ferreira RM, Martins WA, Sposito AC, Halpern B, Saraiva JFK, Carvalho LSF, Tambascia MA, Coelho-Filho OR, Bertolami A, Correa Filho H, Xavier HT, Faria-Neto JR, Bertolami MC, Giraldez VZR, Brandão AA, Feitosa ADM, Amodeo C, Souza DSM, Barbosa ECD, Malachias MVB, Souza WKSB, Costa FAA, Rivera IR, Pellanda LC, Silva MAM, Achutti AC, Langowiski AR, Lantieri CJB, Scholz JR, Ismael SMC, Ayoub JCA, Scala LCN, Neves MF, Jardim PCBV, Fuchs SCPC, Jardim TSV, Moriguchi EH, Schneider JC, Assad MHA, Kaiser SE, Lottenberg AM, Magnoni CD, Miname MH, Lara RS, Herdy AH, Araújo CGS, Milani M, Silva MMF, Stein R, Lucchese FA, Nobre F, Griz HB, Magalhães LBNC, Borba MHE, Pontes MRN, Mourilhe-Rocha R. Atualização da Diretriz de Prevenção Cardiovascular da Sociedade Brasileira de Cardiologia - 2019. Arq Bras Cardiol 2019; 113(4):787-891. 
20. Goff Junior DC, Lloyd-Jones DM, Bennett G, Coady S, D'Agostino RB, Gibbons R, Greenland P, Lackland DT, Levy D, O’Donnell CJ, Robinson JG, Schwartz JS, Shero ST, Smith Junior SC, Sorlie P, Stone NJ, Wilson PWF. American College of Cardiology/American Heart Association Task Force on Practice Guidelines 2013 ACC/AHA guideline on the assessment of cardiovascular risk: a report of the American College of Cardiology/American Heart Association Task Force on Practice Guidelines. J Am Coll Cardiol 2014; 63(25):2935-2959.

21. D’Agostino RB, Vasan RS, Pencina MJ, Wolf PA, Cobain M, Massaro JM, Kannel WB. General cardiovascular risk profile for use in primary care: the Framingham Heart Study. Circulation 2008; 117(6):743-753.

22. Conroy RM, Pyörälä K, Fitzgerald AP, Sans S, Menotti A, De Backer G, De Bacquer D, Ducimetière P, Jousilahti P, Keil U, Njølstad I, Oganov R G, Thomsen T, Tunstall-Pedoe H, Tverdal A, Wedel H, Whincup P, Wilhelmsen L, GrahamIM, SCORE project group. Estimation of ten-year risk of fatal cardiovascular disease in Europe: the SCORE project. Eur Heart J 2003; 24(11):987-1003.

23. Lim SS, Gaziano TA, Gakidou E, Reddy KS, Farzadfar F, Lozano R, Rodgers A. Prevention of cardiovascular disease in high-risk individuals in low-income and middle-income countries: health effects and costs. Lancet 2007; 370(9604):2054-2062.

24. World Health Organization (WHO). World Health organization/International Society of Hypertension (WHO/ISH) risk prediction charts for 14 WHO epidemiological sub-regions. Genebra: WHO; 2007.

25. Allan, GM, Nouri F, Korownyk C, Kolber MR, Vandemeer B,McCormack J. Agreement Among Cardiovascular Disease Risk Calculators. Circulation 2013 127:1948-1956.

26. DeFilippis AR, Young R, Carrubba CJ, McEvoy JW, Budoff MJ, Blumenthal RS, Kronmal RA, McClelland RS, Nasir K, Blaha MJ. An analysis of calibration and discrimination among multiple cardiovascular risk scores in a modern multiethnic cohort. Ann Intern Med 2015; 162(4):266-275.

27. Kavousi M, Leening MJG, Nanchen D, Greenland P, Graham IM, Steyerberg EW, Ikram MA Stricker BH, Hofman A, Franco OH. Comparison of Application of the ACC/AHA Guidelines, Adult Treatment Panel III Guidelines, and European Society of Cardiology Guidelines for Cardiovascular Disease Prevention in a European Cohort. JAMA 2014; 311(14):1416-1423.

28. Sanchez EEM, Urrutia SA, Rodriguez AA, Duarte G, Murillo A, Rivera R, Henriquez AAP, Sanchez DMM, Ordoñez E, Norwood DA, Dominguez LB, Dominguez RL, Torres K, Fajardo EMR, Godoy CA. Cardiovascular risk assessment in the resource limited setting of Western Honduras: An epidemiological perspective. IJC Heart \& Vasculature 2020; 27:100476.

29. The Lancet Statins for millions more? Lancet 2014; 383(9918):669.
30. Ribeiro RA, Duncan BB, Ziegelmann PK, Stella SF Vieira JLC, Restelatto LMF, Polanczyk CA. Cost-effectiveness of high, moderate and low-dose statins in the prevention of vascular events in the Brazilian public health system. Arq Bras Cardiol 2015; 104(1):32-44.

31. Dalton JE Perzynski AT, Zidar DA Rothberg MB, Coulton CJ, Milinovich AT, Einstadter D' Karichu JK, Dawson NV. Accuracy of Cardiovascular Risk Prediction Varies by Neighborhood Socioeconomic Position: A Retrospective Cohort Study. Ann Intern Med 2017; 167(7):456-464.

32. Sivapalaratnam S, Boekholdt SM, Trip MD, Sandhu MS, Luben R, Kastelein JJP, Wareham NJ, Khaw KT. Family history of premature coronary heart disease and risk prediction in the EPIC-Norfolk prospective population study. Family history of premature coronary heart disease and risk prediction in the EPICNorfolk prospective population study. Heart 2010; 96(24):1985-1989.

33. Jackson R, Lawes CMM, Bennett DA, Milne RJ, Rodgers A. Treatment with drugs to lower blood pressure and blood cholesterol based on an individual's absolute cardiovascular risk. Lancet 2005; 365(9457):434441.

34. Lloyd-Jones DM , Hong Y, Labarthe D, Mozaffarian D , Appel LJ , Horn LV, Greenlund K, Daniels S , Nichol D, Tomaselli GF, Arnett DK, Fonarow GC, Ho PM , Lauer MS, Masoudi FA, Robertson RM, Roger V, Schwamm LH, Sorlie P, Yancy CW, Rosamond WD, American Heart Association Strategic Planning Task Force and Statistics Committee. Defining and Setting National Goals for Cardiovascular Health Promotion and Disease Reduction. Circulation 2010; 121(4):586-613.

35. Szwarcwald CL, Malta DC, Pereira CA, Figueiredo AW, Almeida WS, Machado IE, Bacal NS, Silva AG, Silva Júnior JB, Rosenfeld LG. Valores de referência para exames laboratoriais de colesterol, hemoglobina glicosilada e creatinina da população adulta brasileira. Rev Bras Epidemiol 2019; 22(Supl. 2):E190002.

Artigo apresentado em 18/01/2021

Aprovado em 18/01/2021

Versão final apresentada em 20/01/2021

Editores-chefes: Maria Cecília de Souza Minayo, Romeu Gomes, Antônio Augusto Moura da Silva 
